\section{Pupil dilation and driving}

The increasing number of the population now driving motor vehicles means that many patients attending for outpatient visits expect to be able to drive to and from their appointment. This minimises the need for others such as relatives and taxi services to convey patients to hospital and allows them to keep their independence.

Many eye departments, however, issue instructions to patients attending outpatient departments advising that they should not drive themselves to the appointment as they may have drops instilled in their eyes. This may prevent them from driving safely home. Dilation of the pupils forms an integral part of the ocular examination and is necessary if retinal and vitreous pathology is not to be missed. The drops used (commonly tropicamide $1 \%$ ) produce mydriasis and some degree of cycloplegia lasting up to several hours after instillation.

There is precious little evidence that dilation of the pupils per se has any effect on the ability to drive a motor vehicle safely. Several studies have shown that most, but not all, patients retain the same visual acuity after pupil dilation as before $e^{1-3}$ and are therefore able to fulfil the legal requirement of reading a car number plate at $20.5 \mathrm{~m}$ in good light after the examination is over. This equates to an approximate Snellen equivalent of 6/9-2. ${ }^{4}$ High hypermetropes who partially accommodate for distance vision may be compromised. The optical aberrations produced by a large pupil may, however, cause problems with glare and dazzle, especially in adverse driving conditions. Early lens opacities, common in patients attending eye clinics, may maximise these problems. In addition, patients with early macular disease may have prolonged visual acuity reduction after pupil dilation following examination with bright lights because of the photostress effect. Visual acuity, of course, is not the only sensory parameter required for safe driving and the ability to drive is a highly complex task reliant on large numbers of higher perceptual functions. Because of the lack of evidence as to the effect of pupil dilation on driving ability, no national guidelines have been issued either by the DVLA or the Royal College of Ophthalmologists.

The excellent article by Potamitis et al. in this issue $^{5}$ examines some of the visual functions which may be impaired by driving with dilated pupils. As may be expected, visual fields were unimpaired by pupillary dilation, as was the Useful Field of View (UFOV) test, which is a novel technique of assessing visual attention within the driver's peripheral field. Contrast sensitivity, however, was affected, as well as some visual acuity functions to a lesser extent. Of great interest was that drivers on a driving simulator were aware of their visual impairment and altered their driving behaviour appropriately by slowing down and steering more accurately. Unfortunately, the study did not include susceptibility to glare, which does form an important component in the ability to drive, especially at night.

Eye doctors find themselves in a difficult position when it comes to offering advice to their driving patients with dilated pupils. Undoubtedly there is evidence to suggest that some visual functions are affected by pupil dilation. How these would affect individual patients driving home at differing times of the day and with differing road conditions is still unclear. It is important to stress that insurance companies would have reservations about supporting the insured driver if he or she had been involved in a road accident whilst driving with dilated pupils, even if he or she was proven to fulfil the legal visual acuity requirements. ${ }^{6}$ Whilst the Disability and Discrimination Act of 1995 does state that insurers will cover clients who meet the licensing requirements, insurers can apply a temporary loading or increased excess while a person is adapting to a recently acquired disability or condition. ${ }^{7}$

It can be argued that drivers who are aware of a problem with their vision change their driving behaviour accordingly. Many patients with cataracts, for instance, whilst although not prevented by the cataracts from reading the number plate at the legal distance, will either not drive at all or limit themselves to short distances in daylight hours. Despite this selflimitation, it is important that all eye doctors should alert their patients to the fact that pupillary dilatation may have some effect on their ability to drive. We cannot, however, compel the patient not to drive. It is down to the driver, and not the doctor, to be responsible for his or her own actions. Nonetheless; it is important for the doctor to offer some advice.
S. Keightley

North Hampshire Hospital Basingstoke, UK

and

Chairman of the Secretary of State for Transport's Honorary Advisory Panel on Vision and Driving

Simon Keightley, FRCS, FRCOphth

Department of Ophthalmology North Hampshire Hospital Basingstoke, UK 
Although several studies do indicate that pupil dilation has a minimal effect on visual function, especially in young, healthy individuals with emmetropia or low ametropia, many patients attending eye clinics are not typical of this group. We as eye doctors have to be aware of the lowest common denominator amongst our patients and advice that dilating drops may affect the patient's driving ability should still stand.

\section{References}

1. Montgomery DM, MacEwan CJ. Pupil dilatation with tropicamide: the effects on acuity, accommodation and refraction. Eye 1989;3:845-8.
2. Jude EB, Ryan B, O'Leary BM, Gison JM, Dodson PM. Pupillary dilation and driving in diabetic patients. Diabetic Med 1988;15:143-7.

3. Watts P, O'Duffy D, Riddell C, McLeod S, Watson SL. Can I drive after those drops, doctor? Eye 1998;12:963-6.

4. Drasdo N, Haggarty CM. A comparison of British number plates and Snellen vision tests for car drivers. Research report RF676.1981. Crowthorne: Transport and Road Research Laboratory.

5. Potamitis T, Slade SV, Fitt AW, McLaughlin J, Mallen E, Auld RJ, Dunne MCM, Murray PI. The effect of pupil dilation with tropicamide on vision and driving simulator performance. Eye 2000;14:302-6.

6. Fawell D. Personal communication. Association of British Insurers.

7. Association of British Insurers. A guide on insurance for the disabled. 